\title{
Clinical Research Progress on Physical Rehabilitation Therapy for Migraine
}

\author{
Yalun Feng ${ }^{1}$, Sujuan Zhang ${ }^{2}$, Yongmei Yan ${ }^{3 *}$ \\ ${ }^{1}$ Shaanxi University of Traditional Chinese Medicine, Xianyang 712046, Shaanxi Province, China \\ ${ }^{2}$ Ningxia Medical University, Yinchuan 750004, Ningxia Hui Autonomous Region, China \\ ${ }^{3}$ Affiliated Hospital of Shaanxi University of Chinese Medicine, Xianyang 712000, Shaanxi Province, China \\ *Corresponding author: Yongmei Yan, 13609216551@163.com
}

\begin{abstract}
Migraine is a common and recurrent chronic disorder. Migraine is often associated with anxiety, depression and other emotional diseases. With the development of physical rehabilitation techniques, the long-term clinical efficacy of rehabilitation in the treatment of migraine and the prevention of recurrence have been widely accepted. This paper reviews the effectiveness of physical rehabilitation therapy in clinical treatment of migraine in recent years.
\end{abstract}

Keywords: Migraine; Rehabilitation treatment; Transcranial magnetic stimulation; Transcranial electrical stimulation; Acupuncture therapy

Publication date: July 2021; Online publication: July 31, 2021

\section{Introduction}

Migraine is a common chronic neurovascular disease, accompanied by paroxysmal and repeated attacks, that are prolonged and difficult to heal. The prevalence of migraine is $3.3 \%$ to $32.6 \%$ for women and $0.7 \%$ to $16.1 \%$ for men ${ }^{[1]}$. Migraine has become the sixth most disabling disease in the world ${ }^{[2]}$. At this stage, clinicians often choose physical rehabilitation therapy for treatment of migraine as this type of therapy has with short treatment time, quick effect, and simple operation. It does not have the effect on drug metabolism in the body, and it is highly recommended. Under the guidance of clinical guidelines, we should make full use of a variety of physical rehabilitation therapies together with the use medications for treating migraine ${ }^{[3]}$.

\section{Clinical study of transcranial magnetic stimulation for migraine}

Transcranial magnetic stimulation technology is a painless and non-invasive green treatment method. Magnetic signals can penetrate the skull and stimulate the brain nerves without attenuation ${ }^{[4]}$. Azza et al. purposefully evaluated the preventive therapeutic effects of low-frequency repetitive transcranial magnetic stimulation (rTMS) on migraine (with and without aura) and its correlation with the level of serum inflammatory biomarkers (neurokinin A) ${ }^{[5]}$. The study found that the pain intensity, attack frequency, attack duration and migraine disability score of patients with migraine were significantly reduced after transcranial magnetic stimulation treatment, and the patient's serum neurokinin A level was also significantly reduced. This suggests that low frequency rTMS is an effective method to prevent migraine with and without aura. Then, regarding whether the patient is tolerant to transcranial magnetic stimulation, Mohamad et al. conducted a randomized, double-blind, sham-controlled trial to evaluate the effectiveness of repeated transcranial magnetic stimulation in the prevention and treatment of paroxysmal migraine in migraine subjects, and the patient's tolerance to rTMS was evaluated ${ }^{[6]}$. It showed that the 
monthly migraine frequency of patients after rTMS was significantly reduced, and it was found that the activation of the dorsolateral prefrontal cortex (DLPFC) returned to an almost normal level. Based on neuronavigation, fMRI-guided neuronavigation technology is used to target the left motor cortex for target treatment ${ }^{[7]}$. The results showed that the average visual analog scale score, headache frequency, and migraine disability assessment questionnaire in the REAL rTMS group were significantly reduced, and remained unchanged after 1 month of follow-up. It can be seen that 10 fMRI-based rTMS treatments on the left motor cortex may provide long-term pain relief for migraine patients, but further research is needed to confirm the preliminary findings, which gives us a clinical target and provides new ideas for migraine treatment.

\section{Clinical study of transcranial direct current stimulation for migraine}

Transcranial direct current stimulation is a non-invasive technique that uses constant, low-intensity direct current to regulate neuronal activity in the cerebral cortex ${ }^{[8]}$. In a pilot double-blind randomized pseudo-controlled trial, DeIcco et al. studied the treatment of chronic migraine and drug-overdue headache by anode transcranial direct current stimulation, it was found that transcranial direct current stimulation has a certain auxiliary effect on detoxification in patients with chronic migraine and drug-overhead headache [9]. One effect may be closely related to the underlying changes in the cortex-thalamus connection. Pohl et al. explored the long-term effects of transcranial direct current stimulation in preventing paroxysmal migraine ${ }^{[10]}$. This study provides class II evidence for the prevention of episodic migraine by using transcranial direct current stimulation to the visual cortex in patients with episodic migraine, which significantly reduces the number of migraine days per month. Grazzi et al. conducted a double-blind randomized clinical trial to investigate the efficacy of transcranial direct current stimulation in patients with chronic migraine after overdose ${ }^{[11]}$. Studies have shown that transcranial direct current stimulation has no effect on the short-term and long-term course of chronic migraine overdose after acute drug withdrawal, but behavioral education measures for clinicians and support for patient pain management can provide long-term improvement and low recurrence rate.

\section{Clinical research on treatment of migraine with acupuncture of traditional Chinese medicine}

Studies have shown that acupuncture has the same effect as other drugs, which can reduce the frequency of migraine attacks, improve cerebral blood flow, reduce the anxiety of patients, and improve the quality of life of migraine patients ${ }^{[12]}$. Tastan et al. believe that acupuncture can be used as an independent treatment for migraine ${ }^{[13]}$. Linde et al. searched for the clinical trials registered with the World Health Organization and compared acupuncture interventions and non-acupuncture controls in patients with paroxysmal migraine. The results showed that in symptomatic treatment, acupuncture therapy can reduce the incidence of headaches. For patients receiving this treatment, acupuncture can be considered a treatment option ${ }^{[14]}$. In a study of 40 patients with migraine without aura, Qin et al. found that stimulating headache points with balance needles had analgesic effects. Pressing acupuncture is also a common treatment in clinical practice ${ }^{[15]}$. In a study on scalp acupuncture treatment, Liu et al. found that there are a set of acupuncture points in the scalp hairline that are arranged in an orderly manner and highly consistent with the meridian course ${ }^{[16]}$. Certain diseases can be treated by acupuncture on the scalp hairline area, including migraine and insomnia. Peng compared the curative effect of abdominal acupuncture and traditional acupuncture, and concluded that abdominal acupuncture is effective in both acute treatment and preventive treatment of migraine, and the preventive and therapeutic effect of migraine may be better than traditional acupuncture ${ }^{[17]}$.

\section{Prospects}


The physical rehabilitation for treating migraine is simple, convenient, and low-cost. It has attracted the attention and been valued by many clinicians, and has become a common treatment method in clinical 
diagnosis and treatment. Physical rehabilitation therapy is worthy of promotion for clinical use because of its timeliness and practicality, and the fact that it can be accepted by patients owing to its therapeutic role without involving the body's liver and kidney metabolic pathways. However, at present, the number of samples in such studies is small, random allocation is uneven, and most of them are still under clinical observation. Few studies on pathways or targets have been performed. This is not conducive to the promotion and application of non-drug therapies. Thus, we should use this to achieve standardization and internationalization.

\section{Disclosure statement}

The author declares no conflict of interest.

\section{References}

[1] Cady RK, Kathleen F, 2016, Managing Migraine by Patient Profile: Role of Frovatriptan. Patient Preference \& Adherence, 10: 501-510.

[2] Peter JG, Holland PR, 2018, Migraine Therapy: Current Approaches and New Horizons. Springer International Publishing, 15(2): 271-273.

[3] Li SW, Li YS, Liu RZ, et al., 2011, Guidelines for Diagnosis and Treatment of Migraine in China, Chinese Journal of Pain Medicine, 17(2):65-86.

[4] Olejarczyk E, Valiulis V, Germanavicius A, 2021, Effect of Repetitive Transcranial Magnetic Stimulation on Fronto-Posterior and Hemispheric Asymmetry in Depression. Biomedical Signal Processing and Control, 68: 102585.

[5] Hammad AB, Elsharkawy RE, 2021, Abdel Azim GS, Repetitive Transcranial Magnetic Stimulation as a Prophylactic Treatment in Migraine. The Egyptian Journal of Neurology, Psychiatry and Neurosurgery, 57: 5.

[6] Mohamad Safiai NI, Amir NA, Basri H, et al., 2020, Effectiveness and Tolerability of Repetitive Transcranial Magnetic Stimulation for Preventive Treatment of Episodic Migraine: A Single-Centre, Randomised, Double-Blind, Sham-Controlled Phase 2 Trial (Magnet-EM). Trials, 21(1): 923-923.

[7] Kumar A, Mattoo B, Bhatia R, 2021, Neuronavigation Based 10 Sessions of Repetitive Transcranial Magnetic Stimulation Therapy in Chronic Migraine: An Exploratory Study. Neurol Sci, 42(1): 131-139.

[8] Yang YB, Xiao N, Li MY, et al., 2011, Comparison of Transcranial Magnetic Stimulation and Transcranial Direct Current Stimulation. Chinese Rehabilitation Theory and Practice, 17(12): 1131-1135.

[9] De Icco R, Putortì A, De Paoli I, et al., 2021, Anodal Transcranial Direct Current Stimulation in Chronic Migraine and Medication Overuse Headache: A Pilot Double-Blind Randomized Sham-Controlled Trial. Clin Neurophysiol, $\quad 132(1)$ : 126-136. https://doi.org/10.1016/j.clinph.2020.10.014

[10] Pohl H, Moisa M, Jung HH, et al., 2021, Long-Term Effects of Self-Administered Transcranial Direct Current Stimulation in Episodic Migraine Prevention: Results of a Randomized Controlled Trial. Neuromodulation, 24(5): 890-898. https://doi.org/10.1111/ner.13292 
[11] Grazzi L, Usai S, Bolognini N, 2020, No Efficacy of Transcranial Direct Current Stimulation On Chronic Migraine With Medication Overuse: A Double Blind, Randomised Clinical Trial. 
Cephalalgia, 40(11): 1202-1211. https://doi.org/10.1177/0333102420931050

[12] Liu LM, Wang XN, Zhao DX, 2021, Research Status and Progress of Non-Drug Therapy of Traditional Chinese Medicine for Treatment of Migraine. Jilin Journal of Traditional Chinese Medicine, 41(2): 278-280.

[13] Tastan K, Disci OO, Set T, 2018, A Comparison of the Efficacy of Acupuncture and Hypnotherapy in Patients with Migraine. International Journal of Clinical and Experimental Hypnosis, 66(4): 371-385.

[14] Linde K, Allais G, Brinkhaus B, et al., 2016, Acupuncture for the Prevention of Episodic Migraine. The Cochrane Database of Systematic Reviews, 2016(6): CD001218.

[15] Qin XL, Wang WY, Wang JZ, et al., 2019, Analysis on Regional Homogeneity of resting Brain During Balance Acupuncture-Induced Analgesic Effect in Migraine Patients Without Aura. Acupuncture Research, 44(6).

[16] Liu Z, Niu W, 2010, Micro-needling System for Scalp and Hairline. Chinese Acupuncture and Moxibustion, 30(6): 522-524.

[17] Peng A, 2016, Observation on the Clinical Efficacy of Abdominal Acupuncture in the Treatment of Migraine Without Aura. Guangzhou University of Traditional Chinese Medicine. 